\title{
$\mathrm{LiCoO}_{2}$ 电极/电解液界面特性的电化学阻抗谱研究
}

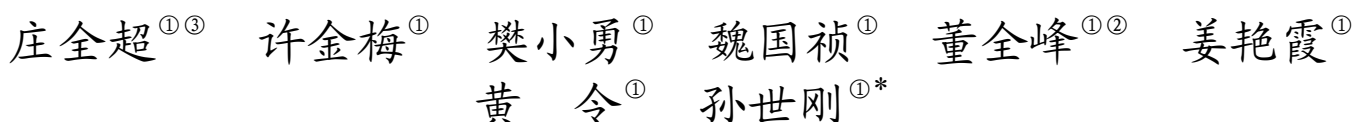

(1) 固体表面物理化学国家重点实验室, 厦门大学化学化工学院化学系, 厦门 361005; (2) 厦门大学宝龙电池研究所,

厦门 361005; (3)西北核技术研究所, 西安 710024)

\begin{abstract}
摘要运用电化学阻抗谱研究了 $\mathrm{LiCoO}_{2}$ 电极在电解液中的财存和首次脱锂过程. 发现 $\mathrm{LiCoO}_{2}$ 电极在电解液中, 随浸泡时间延长其表面 SEI膜不断增厚, 归结为 $\mathrm{LiCoO}_{2}$ 电极与电解液之间的自 发反应导致生成一些高介电常数的有机碳酸锂化合物. 研究结果指出 $\mathrm{LiCoO}_{2}$ 电极首次脱锂过程 中, SEI 膜在 $3.8 \sim 3.95 \mathrm{~V}$ 电位区间发生可逆坉塌, 对应其可逆溶解; 由于过充反应, 当电位大于 $4.2 \mathrm{~V}$ SEI 膜迅速增厚. 研究结果同时表明, $\mathrm{Li} / \mathrm{LiCoO}_{2}$ 电池体系的感抗来源于充放电过程中 $\mathrm{LiCoO}_{2}$ 电极中存在 $\mathrm{LiCoO}_{2} / \mathrm{Li}_{1-x} \mathrm{CoO}_{2}$ 局域浓差电池. 发现锂离子在 $\mathrm{LiCoO}_{2}$ 电极中的嵌脱过程可 较好地用 Langmuir 嵌入等温式和 Frumkin 嵌入等温式描述, 测得 $\mathrm{LiCoO}_{2}$ 电极中锂离子嵌脱过程 中电荷传递反应的对称因子 $\alpha=0.5$.
\end{abstract}

\section{关键词锂离子电池 $\mathrm{LiCoO}_{2}$ 电化学阻抗谱 SEI 膜 感抗}

关于锂电池中锂电极/电解液界面存在化学反应 导致电解液组分发生分解的认识始于 20 世纪 60 年代, 随后人们进一步提出这些还原反应的产物在锂电极 和阴极极化的石墨电极表面形成一层表面膜, 通常 称为“针化膜”或“SEI膜”(solid electrolyte interphase, 固体电解质相界面膜 $)^{[1 \sim 3]}$. SEI膜对锂电池充放电循 环中发生的电化学过程有着重要的影响, 引起了广 泛的兴趣和研究 $[4 \sim 7]$.

SEI膜通常被认为只发生在锂电池的负极, 如锂 电极或石墨电极, 对正极的界面反应研究较少. 最近 几年越来越多的研究工作指出 ${ }^{[8,9]}$, 在正极表面同样 会形成SEI膜，而且正极表面形成的SEI膜同样影响 电池的充放电性能, 虽然它可阻止电解液组分在正 极表面进一步发生分解, 但也增加了正极/电解液界 面的阻抗. Aurbach等人 ${ }^{[10]}$ 认为, $\mathrm{LiCoO}_{2}$ 电极在长期 充放电循环中, 虽然活性材料的失活(degradation)会
逐渐导致电池容量的衰减, 但并不是锂离子电池容 量衰减的主要原因, 限制锂离子电池循环寿命的最 重要因素是电极界面阻抗的增加. Liu等人 ${ }^{[11]}$ 最近报 道, 运用XRD对包覆 $\mathrm{Al}_{2} \mathrm{O}_{3}$ 前后 $\mathrm{LiCoO}_{2}$ 电极性能进行 研究的结果表明, $\mathrm{Al}_{2} \mathrm{O}_{3}$ 包覆的 $\mathrm{LiCoO}_{2}$ 电极与裸 $\mathrm{LiCoO}_{2}$ 电极在充放电过程中几乎都经历了同样的相 变过程, 但 $\mathrm{Al}_{2} \mathrm{O}_{3}$ 包覆的 $\mathrm{LiCoO}_{2}$ 电极即使充电至 4.7 $\mathrm{V}$ 仍能保持较好的结构可逆性. 因此锂离子电池的正 极材料如 $\mathrm{LiCoO}_{2}, \mathrm{LiNiO}_{2}$ 和 $\mathrm{LiMn}_{2} \mathrm{O}_{4}$ 等的电化学性质 在一定程度上与碳负极相似, 强烈地依赖于它们的 表面化学特性.

与锂离子电池碳负极表面SEI膜不同的是, 正极 材料表面往往存在初始的SEI膜, 在正极与电解液接 触过程中 (无外加电压), 初始SEI膜以及正极活性材 料本身均会自发与电解液发生反应, 导致SEI膜的组 成和厚度发生改变. 而且在随后的充放电循环过程 
中SEI膜还会进一步变化 ${ }^{[12 ~ 16]}$. 类似对锂电极或石墨 电极/电解液界面现象的早期研究, 迄今报道的对正 极/电解液界面的研究工作多集中在运用光谱学方法 表征SEI膜的组成和推测其形成机制. 电化学阻抗谱 (EIS) 是研究电极/电解质界面发生的电化学过程的最 有力工具之一，已被广泛地应用于石墨负极表面SEI 膜成膜过程的表征，它也应该能够表征正极表面SEI 膜的成膜过程 ${ }^{[12]}$, 尤其是正极表面SEI膜自发反应成 膜的过程，但这一工作迄今未见文献报道. 此外，在 文献中的研究工作多采用为特定目的自行制备的电 极, 它与商品化锂离子电池中的电极在组成和制备 条件方面往往存在一定的差异. 在本文中, 为此我们 按照商品化锂离子电池 $\mathrm{LiCoO}_{2}$ 正极的组成和制备条 件制备了 $\mathrm{LiCoO}_{2}$ 电极, 运用EIS研究其在电解液中的 咜存和首次脱锂过程，重点探讨SEI膜的成膜机制以 及感抗产生的原因.

\section{1 实验部分}

实验在自制三电极玻璃电解池中完成, 金属锂 片作为参比和辅助电极, $\mathrm{LiCoO}_{2}$ 电极按 $92 \%$ 的活性材 料(天津巴莫科技股份有限公司)、 $5 \%$ 的 PVDF-HFP 粘合剂(Kynar FLEX LBG, Elf-atochem, USA)和 3\%的 导电碳黑(上海杉杉科技有限公司)的质量百分比组成, 在厦门大学宝龙电池研究所制备. 电解液组成为 1 $\mathrm{mol} \cdot \mathrm{L}^{-1} \mathrm{LiPF}_{6}-\mathrm{EC}($ 碳酸乙烯酯)：DEC(碳酸二乙酯)： $\mathrm{DMC}($ 碳酸二甲酯)(体积比, $1: 1: 1)($ 张家港国泰华 荣化工新材料公司).

EIS 测试在 Par2263-1 型电化学综合测试仪 (Princeton Applied Research, USA)上完成, 测试频率 范围 $10^{5} \sim 10^{-2} \mathrm{~Hz}$, 交流信号振幅 $5 \mathrm{mV}$. 在进行阻抗 测试前, 电极在极化电位平衡 $2 \mathrm{~h}$.

\section{2 结果与讨论}

\section{$2.1 \mathrm{LiCoO}_{2}$ 电极在电解液中贮存和首次脱锂过 程中的 EIS 谱基本特征}

图 1 为 $\mathrm{LiCoO}_{2}$ 电极随电极电位 $E$ 升高其 $E I S$ 谱的 变化. 可以看出, 新制备的 $\mathrm{LiCoO}_{2}$ 电极在电解液中 的开路电位为 $3.5 \mathrm{~V}$, 其EIS (Nyquist图)在整个测试频 率范围内可分为两部分, 即高频区域的小半圆和中 低频区域的一段圆弧. Aurbach等人 ${ }^{[17,18]}$ 指出，高频 区域的半圆与SEI膜有关，中低频区域的圆弧与巨大 的电荷传递电阻和双电层电容有关. 随着 $E$ 的升高, 中低频区域圆弧的曲率半径不断减小, $3.85 \mathrm{~V}$ 时近似 为一个半圆. $3.9 \mathrm{~V}$ 时的EIS 由三部分组成, 即高频区 域与SEI膜有关的半圆(high frequency arc，简称HFA), 中频区域与电荷传递电阻和双电层电容有关的半圆 (middle frequency arc, 简称MFA)以及低频区域反映 锂离子固态扩散过程的斜线. $3.95 \mathrm{~V}$ 时 EIS
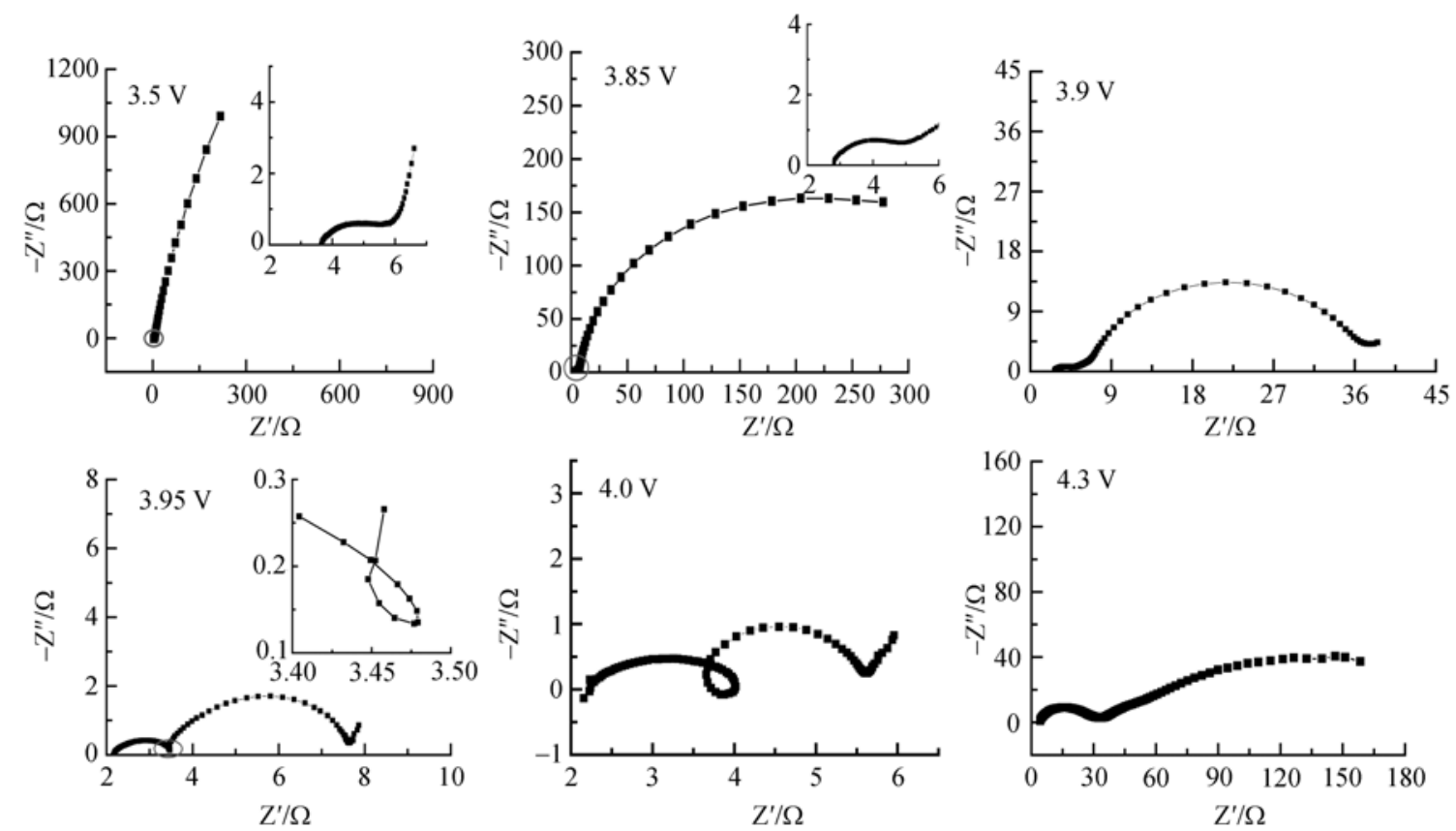

图 $1 \mathrm{LiCoO}_{2}$ 电极首次脱锂过程中 $\mathrm{EIS}$ 随电极电位 $E$ 升高的变化 


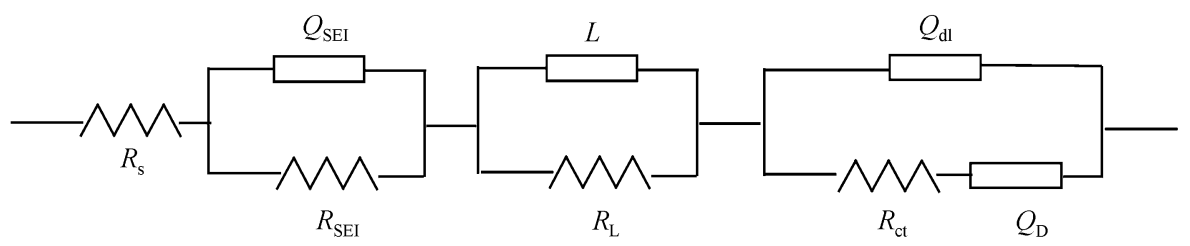

图 $2 \mathrm{LiCoO}_{2}$ 电极在电解液中咜存和首次脱锂过程中 EIS 的等效电路

的一个重要特征就是在中高频区域出现与感抗相关 的半圆(inductive loop, 简称 IL), 至 $4.0 \mathrm{~V}$ 时 EIS 由 4 个完全相互分离的区域组成, 即 HFA, IL, MFA 以及 低频区域斜线. $4.3 \mathrm{~V}$ 时 EIS 中 IL 和低频区的斜线消 失, MFA 再次演变为一段圆弧, 说明此时已不存在锂 离子的脱出过程.

\section{2 等效电路的选取}

根据实验结果我们提出 $\mathrm{LiCoO}_{2}$ 电极在电解液中 足存和首次脱锂过程中 EIS 的等效电路如图 2 所示. 其中 $R_{\mathrm{S}}$ 代表欧姆电阻, $R_{\mathrm{SEI}}, R_{\mathrm{ct}}$ 和 $R_{\mathrm{L}}$ 分别为 $\mathrm{SEI}$ 膜电 阻、电荷传递电阻和与电感对应的电阻, $L$ 为电感, SEI 膜电容 $C_{\mathrm{SEI}}$ 、双电层电容 $C_{\mathrm{d} 1}$ 以及扩散阻抗分别 用恒相角元件(CPE) $Q_{\mathrm{SEI}}, Q_{\mathrm{dl}}$ 和 $Q_{\mathrm{D}}$ 表示. $\mathrm{CPE}$ 的导纳 响应表达式如下:

$$
Y=Y_{0} \omega^{n} \cos \left(\frac{n \pi}{2}\right)+\mathrm{j} Y_{0} \omega^{n} \sin \left(\frac{n \pi}{2}\right),
$$

其中 $\omega$ 为角频率, $\mathrm{j}$ 为虚数单位 $\sqrt{-1}$. 当 $n=0$ 时, CPE 相当于一个电阻; $n=1, \mathrm{CPE}$ 相当于一个电容; $n=0.5$, CPE 相当于 Warburg 阻抗. 在本研究中, 当 $0.5<n<1$ 时, $Y_{0}$ 被当作一个假电容, 其值近似等于电容 $C$ 的值.

图 2 所示的等效电路能较好地拟合不同电位下 获取的 EIS 实验数据. 其典型结果如图 3 所示，拟合 得到的等效电路中各个参数列于表 1 中. 可以看出, 实验数据曲线与拟合曲线实现较好重叠，各等效电 路参数拟合误差一般小于 $15 \%$.

\section{$2.3 \mathrm{LiCoO}_{2}$ 电极在电解液中贮存和首次脱锂过 程中 SEI 膜的生长规律}

图 4 和 5 分别为 $\mathrm{LiCoO}_{2}$ 电极在电解液中浸泡和 首次脱锂过程中, $R_{\mathrm{SEI}}, Q_{\mathrm{SEI}}-n$ 和 $Q_{\mathrm{SEI}}-Y_{0}$ 随浸泡时间 $t$ 和电极电位 $E$ 的变化. 根据 SEI 模型, SEI 膜的电阻 $R_{\mathrm{SEI}}$ 和电容 $C_{\mathrm{SEI}}$ 与 SEI 膜的电阻率 $\rho$ 和介电常数 $\varepsilon$ 的 关系如方程式(2)和(3)所示.

$$
R_{\mathrm{SEI}}=\rho l / S,
$$

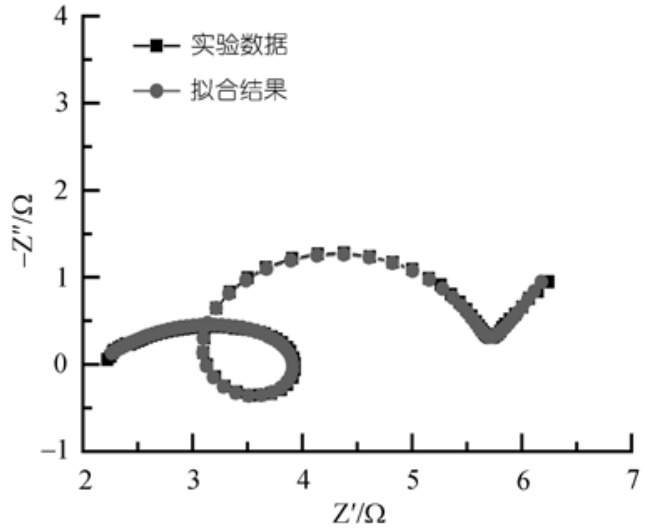

图 $34.05 \mathrm{~V}$ 时 EIS 实验数据与使用图 2 所示等效 电路模拟结果对比

表 $14.05 \mathrm{~V}$ 时 EIS 拟合获得的等效电路参数值

\begin{tabular}{llc}
\hline 等效电路 & \multicolumn{1}{c}{ 数值 } & 拟合误差 \\
\hline$R_{\mathrm{s}} / \Omega$ & 0.83486 & $4.6865 \%$ \\
$R_{\mathrm{SEI}} / \Omega$ & 2.253 & $2.5793 \%$ \\
$Q_{\mathrm{SEI}}-n$ & 0.50358 & $2.505 \%$ \\
$Q_{\mathrm{SEI}}-Y_{0} / \mathrm{F}$ & $3.5728 \times 10^{-3}$ & $12.403 \%$ \\
$R_{\mathrm{ct}} / \Omega$ & 2.572 & $1.8635 \%$ \\
$Q_{\mathrm{dl}}-n$ & 0.96045 & $1.3457 \%$ \\
$Q_{\mathrm{dl}}-Y_{0} / \mathrm{F}$ & $2.422 \times 10^{-2}$ & $2.6441 \%$ \\
$Q_{\mathrm{D}}-n$ & 0.66819 & $3.7617 \%$ \\
$Q_{\mathrm{D}}-Y_{0} / \mathrm{F}$ & 5.929 & $7.5729 \%$ \\
$L / \mathrm{H}$ & $5.7467 \times 10^{-3}$ & $4.1055 \%$ \\
$R_{\mathrm{L}} / \Omega$ & 2.561 & $4.6607 \%$ \\
$\chi^{2}$ & $8.5031 \times 10^{-4}$ & \\
\hline
\end{tabular}

$$
C_{\mathrm{SEI}}=\varepsilon S / l,
$$

式中 $l$ 为 SEI膜的厚度, $S$ 为电极的表面积. 从图 4 可以 看出, $R_{\mathrm{SEI}}$ 随 $t$ 增加不断变大, 但其增长速率逐渐减小. 显示一方面随 $t$ 延长 $\mathrm{LiCoO}_{2}$ 电极表面SEI膜不断增厚; 另一方面 $\mathrm{LiCoO}_{2}$ 电极表面 SEI膜的形成反过来抑制 $\mathrm{LiCoO}_{2}$ 电极与电解液之间的自发反应. $Q_{\mathrm{SEI}}-Y_{0}$ 随 $t$ 延长而增大, 指示 SEI膜介电常数 $\varepsilon$ 随 $t$ 延长而增大, 说明 $\mathrm{LiCoO}_{2}$ 电极和电解液之间的自发反应, 主要生 成一些高介电常数的有机碳酸锂化合物, 这与Wang 等人 ${ }^{[19]}$ 用傅里叶变换红外和拉曼光谱研究的 

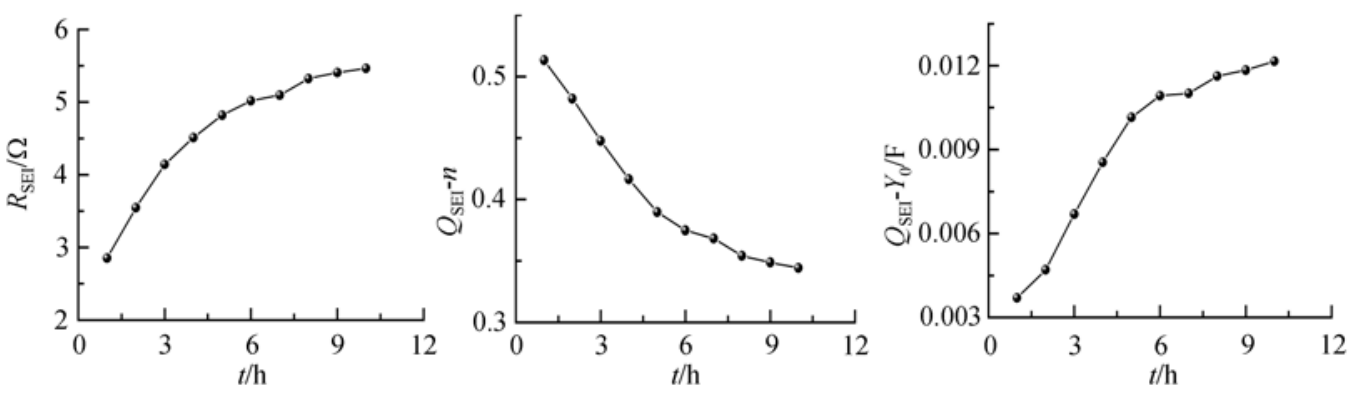

图 $4 \mathrm{LiCoO}_{2}$ 电极在电解液中浸泡过程中, $R_{\mathrm{SEI}}, Q_{\mathrm{SEI}^{-} n}$ 和 $Q_{\mathrm{SEI}^{-}} Y_{0}$ 随浸泡时间 $t$ 延长的变化
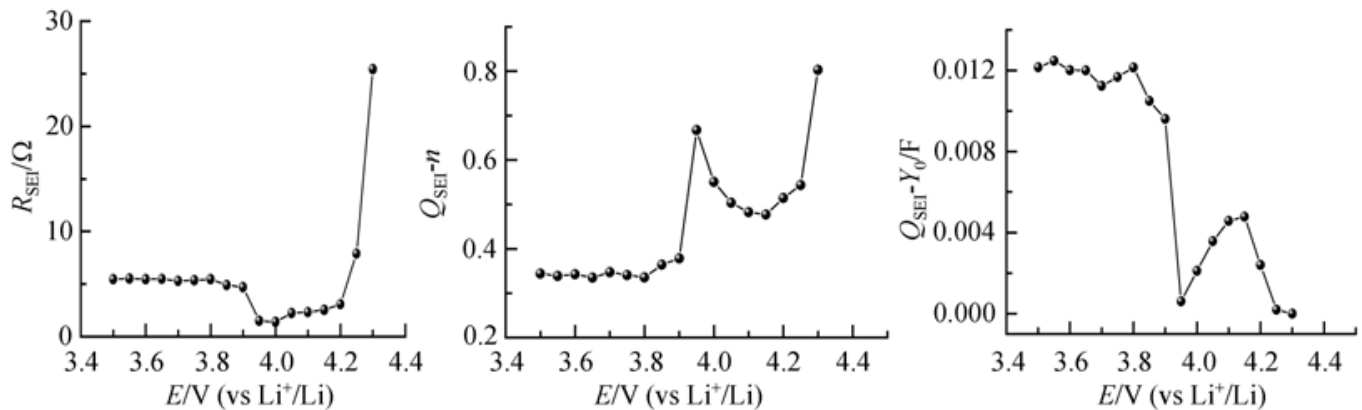

图 $5 \mathrm{LiCoO}_{2}$ 电极在首次脱锂过程中, $R_{\mathrm{SEI}}, Q_{\mathrm{SEI}^{-} n}$ 和 $Q_{\mathrm{SEI}^{-}} Y_{0}$ 随电极电位 $E$ 升高的变化

结果一致. $Q_{\mathrm{SEI}}-n$ 随 $t$ 延长而减小, 对应 SEI 膜均匀程 度降低, 可能与高介电常数的有机碳酸锂化合物的 生成有关. 此外, 随 $t$ 延长至 2 小时后，出现 $Q_{\mathrm{SEI}-n}$ 小于 0.5 , 这可能对应 $\mathrm{LiCoO}_{2}$ 电极表面由于 SEI 膜的 自发成膜变得异常不均匀和粗糙.

图 5 的结果显示, $\mathrm{LiCoO}_{2}$ 电极在首次脱锂过程中, $3.8 \mathrm{~V}$ 以下, 随 $E$ 升高, $R_{\mathrm{SEI}}, Q_{\mathrm{SEI}}-n$ 和 $Q_{\mathrm{SEI}}-Y_{0}$ 基本保持 不变, 表明 $\mathrm{LiCoO}_{2}$ 电极经过在电解液中的长时间浸 泡表面SEI膜已经具有一定的稳定性. 3.8 3.9 V之间, $R_{\mathrm{SEI}}$ 和 $Q_{\mathrm{SEI}}-Y_{0}$ 缓慢减小, $Q_{\mathrm{SEI}}-n$ 缓慢增大; $3.9 \sim 3.95 \mathrm{~V}$ 之间 $R_{\mathrm{SEI}}$ 和 $Q_{\mathrm{SEI}}-Y_{0}$ 急剧减小, $Q_{\mathrm{SEI}}-n$ 急剧增大, 上述现 象一般归因于SEI膜的可逆坡塌, 由于缺乏显微学方面 的证据，产生这一现象的原因和机制有待进一步研究 [8]. 比较图 4 和 $5, \mathrm{LiCoO}_{2}$ 电极在首次脱锂过程中 $R_{\mathrm{SEI}}$, $Q_{\mathrm{SEI}} n$ 和 $Q_{\mathrm{SEI}}-Y_{0}$ 的变化规律与 $\mathrm{LiCoO}_{2}$ 电极在电解液 中浸泡过程中的变化规律相反, 由此推测 SEI膜的可 逆坍塌主要是由于SEI膜的可逆溶解造成. $4.0 \sim 4.15 \mathrm{~V}$ 之间, $R_{\mathrm{SEI}}$ 和 $Q_{\mathrm{SEI}}-Y_{0}$ 缓慢增大, $Q_{\mathrm{SEI}}-n$ 缓慢减小, 这可能 与锂离子脱出过程中 $\mathrm{LiCoO}_{2}$ 电极发生的可逆相变有 关. $4.2 \mathrm{~V}$ 以上, $R_{\mathrm{SEI}}$ 和 $Q_{\mathrm{SEI}}-n$ 快速增大, $Q_{\mathrm{SEI}}-Y_{0}$ 快速减 小, 指示SEI膜快速增厚, 这一现象显然是由于有机溶 剂的过充电氧化造成的, 因此 $\mathrm{LiCoO}_{2}$ 电极的充放电
过程必须严格控制在 $4.2 \mathrm{VW下.}$

\section{$2.4 \mathrm{LiCoO}_{2}$ 电极在充电过程中感抗产生机制分析}

从图 1 可以看出，与感抗相关的半圆(IL)首先出 现在 $3.95 \mathrm{~V}$, 即发生部分锂离子脱出的电位区域. 当 电位高于 $4.3 \mathrm{~V}$ 时, 即锂离子全部脱出后(对应于 $\mathrm{Li}_{1-x} \mathrm{CoO}_{2}, x=0.5$ 时), IL 消失. 电感通常定义为当流 经电路中的电流发生改变时，便会在这一电路中产 生感应电动势阻止电流的变化. 因此对 IL 的合理解 释应该为: IL 是由于锂离子的脱出而产生的感应电动 势引起的. 它产生的原因可能是由于锂离子脱出过 程中的不均衡性，导致不同 $\mathrm{LiCoO}_{2}$ 颗粒之间锂离子 的脱出量不同, 产生被 SEI 膜分隔的富锂和贫锂区域, 从而在被 SEI 膜分隔的 $\mathrm{LiCoO}_{2}$ 和 $\mathrm{Li}_{1-x} \mathrm{CoO}_{2}(0<x<0.5)$ 之间形成局域浓差电池. 因为 SEI 膜并不是完美无缺 的，在锂离子脱出过程中，上述局域浓差电池中的两 电极间就会有局域电流通过，从而产生电场对抗锂 离子脱出产生的电场. 因此这一局域浓差电池在 $\mathrm{LiCoO}_{2}$ 电极充电过程中产生阻止锂离子从 $\mathrm{LiCoO}_{2}$ 电 极中脱出的感应电流，上述情况符合感抗产生的条 件. 为了更好地认识 $\mathrm{Li} / \mathrm{LiCoO}_{2}$ 电池体系中存在的 IL, 我们在图 6 中给出 $\mathrm{LiCoO}_{2}$ 电极中 $\mathrm{LiCoO}_{2} / \mathrm{Li}_{1-x} \mathrm{CoO}_{2}$ 


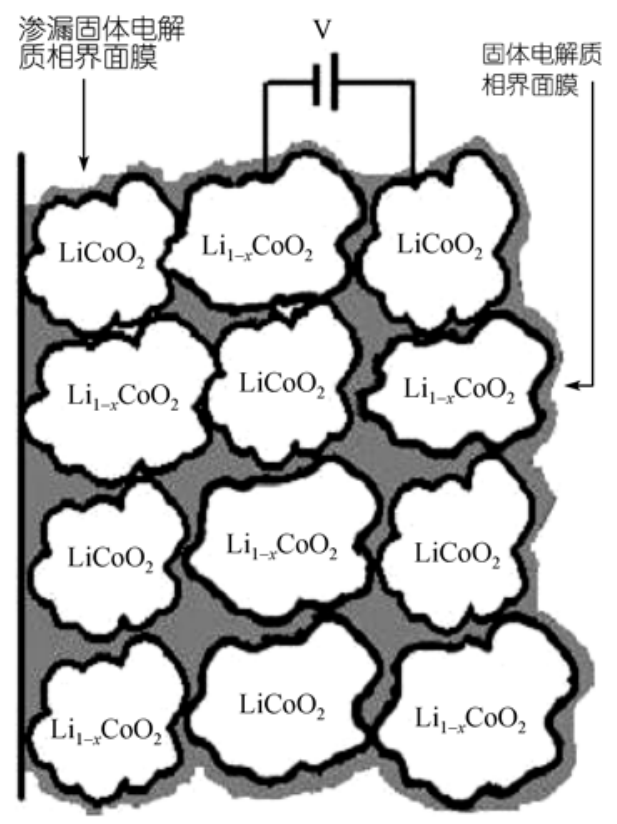

图 $6 \mathrm{LiCoO}_{2}$ 电极在首次脱锂过程中, 局域浓差电池 示模型意图

局域浓差电池模型的示意图. 在 $\mathrm{LiCoO}_{2}$ 电极充电过 程中, 伴随锂离子的脱出 $\mathrm{LiCoO}_{2} / \mathrm{Li}_{1-x} \mathrm{CoO}_{2}$ 局域浓差 电池会不断地渗漏电流, 直至锂离子完全脱出(对应 于 $\mathrm{LiCoO}_{2}$ 全部转变为 $\left.\mathrm{Li}_{0.5} \mathrm{CoO}_{2}\right)$, 即 $\mathrm{LiCoO}_{2}$ 电极内部 不再存在锂离子的浓差极化. 因此 $\mathrm{LiCoO}_{2}$ 电极表面 的这一SEI膜也可称为“渗漏SEI膜”(Leaky SEI). 以上 分析表明，本文提出的 $\mathrm{Li} / \mathrm{LiCoO}_{2}$ 电池体系中感抗的 $\mathrm{LiCoO}_{2} / \mathrm{Li}_{1-x} \mathrm{CoO}_{2}$ 局域浓差电池机制可以较好地解释 所得的实验结果. 值得指出的是, Gnanaraj等人 [20]将 $\mathrm{Li} / \mathrm{C}$ 电池体系中的感抗也归因于存在 $\mathrm{LiC}_{6} / \mathrm{C}_{6}$ 浓差电 池. 根据以上局域浓差电池模型，我们可以预测伴随 锂离子的脱出, 当 $\mathrm{LiCoO}_{2}$ 电极中一半的 $\mathrm{LiCoO}_{2}$ 转变 为 $\mathrm{Li}_{0.5} \mathrm{CoO}_{2}$ 时, 即锂离子的浓差极化达到最大值时, 由 $\mathrm{LiCoO}_{2} / \mathrm{Li}_{1-x} \mathrm{CoO}_{2}$ 局域浓差电池产生的感抗会出现 一个极大值. 图 7 中的实验结果显示 $R_{\mathrm{L}}$ 和 $L$ 随 $E$ 升高出 现一个极大值, 从而证明了在 $\mathrm{Li} / \mathrm{LiCoO}_{2}$ 电池体系中 感抗是由于存在 $\mathrm{LiCoO}_{2} / \mathrm{Li}_{1-x} \mathrm{CoO}_{2}$ 局域浓差电池引 起. 此外，当采用用球磨制浆和涂片的方法制备 $\mathrm{LiCoO}_{2}$ 电极, 由于电极材料混合均匀, 容易达到平 衡, 因此在 EIS 中的感抗消失, 还观察到 $\mathrm{LiCoO}_{2} /$ $\mathrm{Li}_{1-x} \mathrm{CoO}_{2}$ 局域浓差电池充电后随放置时间增加而消 失. 这些实验结果也进一步验证了 $\mathrm{LiCoO}_{2} / \mathrm{Li}_{1-x} \mathrm{CoO}_{2}$ 局域浓差电池是产生感抗的根源.
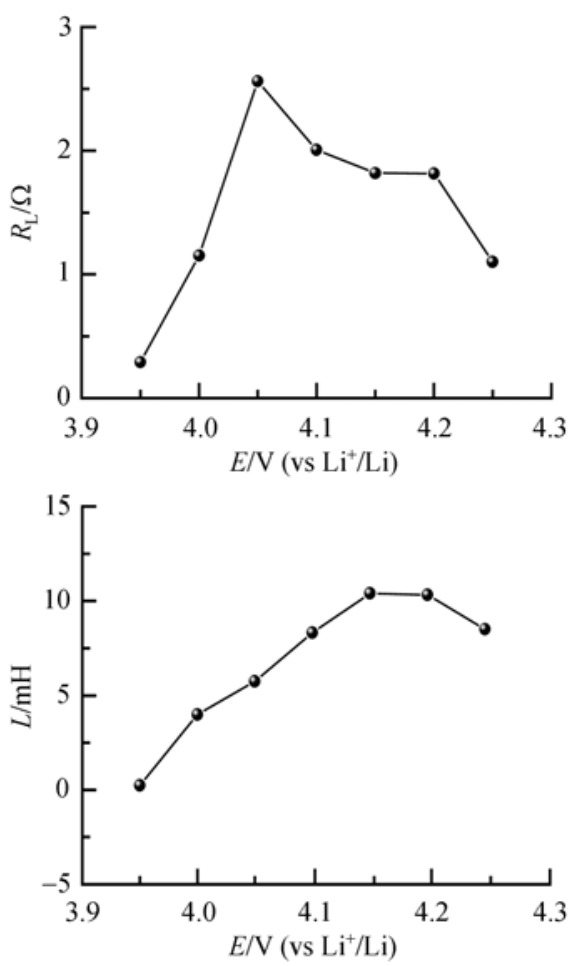

图 $7 \mathrm{LiCoO}_{2}$ 电极在首次脱锂过程中, $R_{\mathrm{L}}$ 和 $L$ 随 $E$ 升高 的变化

\section{5 电荷传递电阻随电极电位变化的分析}

电荷传递电阻 $R_{\mathrm{ct}}$ 的一般表达式如(4)式所示:

$$
R_{\mathrm{ct}}=\frac{1}{\partial I_{\mathrm{f}} / \partial E},
$$

其中 $\partial I_{\mathrm{f}} / \partial E$ 为在稳态条件下, 法拉第电流对电极电 位的偏微分. 众所周知 $[21]$, 对于受阻滞扩散条件 (blocking diffusion conditions) 限制的嵌入过程，稳 态即意味着平衡状态. 因此电荷传递电阻可表示为:

$$
R_{\mathrm{ct}}=\frac{1}{f F A v_{0}},
$$

其中 $f=F / R T$ (式中 $F$ 为法拉第常数, $R$ 为气体常数, $T$ 为热力学温度), $A$ 为具有电活性的电极表面积, $v_{0}$ 为平衡时嵌入反应的交换速度. 因此 $A v_{0}$ 能够通过 (5) 式进行计算. 图 8 为 $\mathrm{LiCoO}_{2}$ 电极在首次脱锂过程中, 实验测得 $\ln R_{\mathrm{ct}}$ 随 $E$ 升高的变化. 可以看出, 在 $4.05 \mathrm{~V}$ 以上的高电位区域 $\ln R_{\mathrm{ct}}$ 与 $E$ 呈线性关系, 即 $v_{0}$ 与 $E$ 呈指数关系, 从而:

$$
v_{0}=k \exp (-\alpha f E) .
$$

从图 8 中的直线斜率计算得到 $\alpha=0.5$, 这一数值 与电化学动力学中电荷传递过程的对称因子基本一 
致. 我们进一步将公式(6)与基于嵌入等温式 (insertion isotherm)的嵌入过程交换速率理论表达式 进行对比. 如果假定锂离子嵌入过程的非理想行为 是由嵌入 $\mathrm{LiCoO}_{2}$ 电极的锂离子之间、锂离子与嵌锂 空位之间的相互作用造成的，则弗鲁姆金等温式 (Frumkin isotherm)可适用于锂离子嵌入 $\mathrm{LiCoO}_{2}$ 电极 的过程 ${ }^{[17,22]}$. 在上述假设下, 交换速率可表示为:

$$
v_{0}=K_{\mathrm{r}} M^{+} c_{T}(1-x) \exp \left(-\alpha_{\mathrm{r}} g x\right)=K_{\mathrm{o}} c_{T} x \exp \left(\alpha_{0} g x\right) \text {, }
$$

式中 $K_{\mathrm{r}}$ 和 $K_{\mathrm{o}}$ 分别为氧化和还原反应的电荷传递速率 常数:

$$
\begin{gathered}
K_{\mathrm{o}}=k_{\mathrm{o}} \exp \left(\alpha_{\mathrm{o}} f E\right), \quad K_{\mathrm{r}}=k_{\mathrm{r}} \exp \left(-\alpha_{\mathrm{r}} f E\right), \\
\alpha_{\mathrm{o}}+\alpha_{\mathrm{r}}=1,
\end{gathered}
$$

其中 $M^{+}$为电解液中锂离子的本体浓度, $c_{\mathrm{T}}$ 为嵌入 $\mathrm{LiCoO}_{2}$ 的锂离子最大浓度. $\alpha_{\mathrm{o}}$ 和 $\alpha_{\mathrm{r}}$ 分别为氧化和还 原反应的对称因子. $g$ 为弗鲁姆金参数, $g>0$ 和 $g<0$ 分别对应嵌入 $\mathrm{LiCoO}_{2}$ 电极的锂离子之间和锂离子与 嵌锂空位之间的相互排斥和吸引作用.

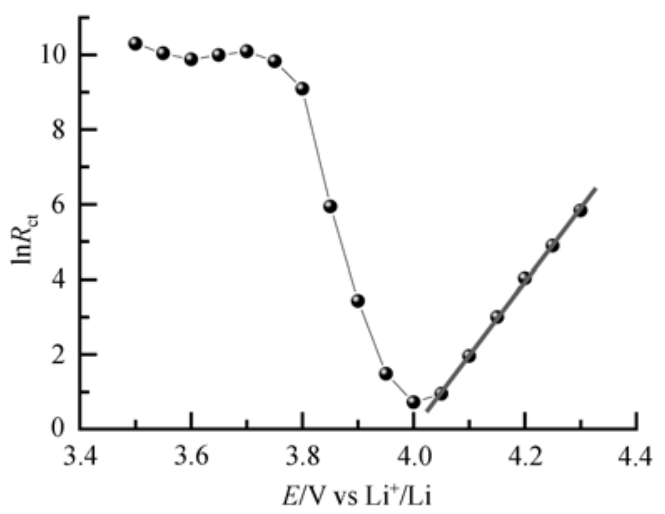

图 $8 \mathrm{LiCoO}_{2}$ 在首次脱锂过程中, $\ln R_{\mathrm{ct}}$ 随 $E$ 升高的变化

假定(7)式中 $g=0$, 即假定不存在嵌入 $\mathrm{LiCoO}_{2}$ 电 极的锂离子之间和锂离子与嵌锂空位之间的相互作 用, 我们可得到 Langmuir 嵌入等温式(Langmuir insertion isotherm). 显然, 公式(6)只是公式(7)的极限 形式, 即在锂离子嵌入量很少的情况下 $(x \rightarrow 0, E \rightarrow$ $\infty)$, 可得出 $k=k_{\mathrm{r}} M^{+} c_{T}$, 以及 $\alpha=\alpha_{\mathrm{r}}$. 从以上讨论可以 得到, 在锂离子嵌入量很少的情况下, Langmuir 嵌入 等温式和 Frumkin 嵌入等温式均可较好地描述锂离 子在 $\mathrm{LiCoO}_{2}$ 电极中的嵌脱过程.

如果同样假定(7)式中 $g=0, M^{+}=1$ 及 $c_{T}=1$, 同时 引入标准交换速率常数 $k_{\mathrm{s}}$ 这一物理量, 从(7)式可得

$$
\begin{aligned}
& v_{0}=k_{\mathrm{s}} x^{0.5}(1-x)^{0.5}, \\
& k_{\mathrm{s}}=k_{\mathrm{r}} \exp \left(-\alpha_{\mathrm{r}} f E_{0}\right)=k_{\mathrm{o}} \exp \left(-\alpha_{\mathrm{o}} f E_{0}\right), \\
& E_{0}=\frac{1}{f} \ln \frac{k_{\mathrm{o}}}{k_{\mathrm{r}}} .
\end{aligned}
$$

将(9)式代入(5)式得

$$
R_{\mathrm{ct}}=\frac{1}{f F A k_{\mathrm{s}} x^{0.5}(1-x)^{0.5}} .
$$

(10)式预示, 当 $x=0.5$ 时 $R_{\mathrm{ct}}$ 存在极小值, 当 $x<0.5$, $R_{\mathrm{ct}}$ 随 $x$ 减小而增大. $x>0.5, R_{\mathrm{ct}}$ 随 $x$ 增大而增大. 可以 看出, 公式(10)能够较好的解释图 8 中 $3.7 \mathrm{~V}$ 以上 $\ln R_{\mathrm{ct}}$ 随 $E$ 升高发生的变化的实验结果. $3.7 \mathrm{~V}$ 以下, $\ln R_{\mathrm{ct}}$ 随 $E$ 升高发生的变化情况与公式(10)不完全符合，可能 与此时 $\mathrm{LiCoO}_{2}$ 电极处于锂离子完全嵌满的状态, 嵌 入 $\mathrm{LiCoO}_{2}$ 电极的锂离子之间和锂离子与嵌锂空位之 间存在强相互作用有关.

\section{3 结论}

本文运用 EIS 研究了 $\mathrm{LiCoO}_{2}$ 电极在电解液中的 咜存和首次脱锂过程. 结果指出, $\mathrm{LiCoO}_{2}$ 电极在电解 液中浸泡过程中，随浸泡时间延长 $\mathrm{LiCoO}_{2}$ 电极表面 SEI 膜不断增厚, $\mathrm{LiCoO}_{2}$ 电极和电解液之间的自发反 应，主要生成一些高介电常数的有机碳酸锂化合物. $\mathrm{LiCoO}_{2}$ 电极在脱锂过程中, $3.8 \sim 3.95 \mathrm{~V}$ 之间发生可 逆坞塌, 归因于 SEI 膜的可逆溶解造成; $4.2 \mathrm{~V}$ 以上由 于过充电反应, SEI 膜迅速增厚. 根据实验结果提出 了在 $\mathrm{Li} / \mathrm{LiCoO}_{2}$ 电池体系中存在的 $\mathrm{LiCoO}_{2} / \mathrm{Li}_{1-x} \mathrm{CoO}_{2}$ 局域浓差电池导致产生感抗的模型, 并由实验结果 得到验证. 对本文实验结果的分析还证实锂离子在 $\mathrm{LiCoO}_{2}$ 电极中的嵌脱过程可较好地用 Langmuir 嵌入 等温式和 Frumkin 嵌入等温式描述, 并测得锂离子在 $\mathrm{LiCoO}_{2}$ 电极中嵌脱过程的电荷传递反应对称因子 $\alpha=$ 0.5 .

\section{参考文献}

1 Thevemin J G, Muller R H. Impedance of lithium electrode in a propylene carbonate electrolyte. J Electrochem Soc, 1987, 134(2): $273-280[\mathrm{DOI}]$

2 Peled E, Goldnitsky D, Ardel G. Advanced model for solid electrolyte interphase of lithium electrode in liquid and polymer electrolytes. J Electrochem Soc, 1997, 144(8): L208－L210[DOI]

3 Peled E. The electrochemical behavior of alkali and alkaline earth metals in nonaqueous battery systems-the solid electrolyte interphase model. J Electrochem Soc, 1979, 126(12): 2047-2051 
4 Aurbach D, Weissman I, Schechter A. X-ray photoelectron spectroscopy studies of lithium surfaces prepared in several important electrolyte solutions. A comparison with previous studies with previous studies by fourier transform infrared spectroscopy. Langmiur, 1996, 12(16): 3991 - 4007[DOI]

5 Schechter A, Aurbach D. X-ray photoelectron spectroscopy study of surface films formed on Li electrodes freshly prepared in alkyl carbonate solutions. Langmuir, 1999, 15(9): 3334-3342[DOI]

6 Jeong $\mathrm{S}$ K, Inaba M, Iriyama Y, Abe T, Ogumi Z. Surface film formation on a graphite negative electrode in lithium-ion batteries: AFM study on the effects of co-solvents in ethylene carbonate-based solutions. Electrochim Acta, 2002, 47(12): 1975-1982 [DOI]

7 Morigaki K-i. In situ analysis of the interfacial reactions between MCMB electrode and organic electrolyte solutions. J Power Sources, 2002, 103(2): 253-264[DOI]

$8 \mathrm{Xu} \mathrm{K}$. Nonaqueous liquid electrolytes for lithium-based rechargeable batteries. Chem Rev, 2004, 104(10): 4303-4418[DOI]

9 Würsig A, Buqa H, Holzapfel M, Krumeich F, Novák P. Film formation at positive electrodes in lithium-ion batteries. Electrochem Solid-State Lett, 2005, 8(1): A34-A37[DOI]

10 Aurbach D, Markovsky B, Rodkin A, Cojocaru M, Elena Levi E, Kim H J. An analysis of rechargeable lithium-ion batteries after prolonged cycling. Electrochim Acta, 2002, 47(12): 1899-1911 [DOI]

11 Liu L, Chen L, Huang X, Yang X, Yoon W S, Lee H S, McBreen J. Electrochemical and in situ synchrotron XRD studies on $\mathrm{Al}_{2} \mathrm{O}_{3}$-coated $\mathrm{LiCoO}_{2}$ cathode material. J Electrochem Soc, 2004, 151(9): A1344-A1351[DOI]

12 Aurbach D, Gamolsky K, Markovsky B, Salitra S, Gofer Y, Heider $\mathrm{U}$, Oesten R, Schmidt M. The study of surface phenomena related to electrochemical lithium intercalation into $\mathrm{Li}_{x} \mathrm{MO}_{y}$ host materials $(\mathrm{M}$ $=\mathrm{Ni}, \mathrm{Mn})$. J Electrochem Soc, 2000, 147(4): 1322-1331[DOI]

13 Eriksson T, Andersson A M, Bishop A G, Gejke C, Gustafsson T, Thomas J O. Surface analysis of $\mathrm{LiMn}_{2} \mathrm{O}_{4}$ electrodes in carbonate-based electrolytes. J Electrochem Soc, 2002, 149(1): A69-

\section{A78[DOI]}

14 Matsuo Y, Kostecki R, Mclarnon F. Surface layer formation on thin-film $\mathrm{LiMn}_{2} \mathrm{O}_{4}$ electrodes at elevated temperatures. J Electrochem Soc, 2001, 148(7): A687-A692[DOI]

15 Ostrovskii D, Ronci F, Acrosati B, Jacobsson P. Reactivity of lithium battery electrode materials toward non-aqueous electrolytes: spontaneous reactions at the electrode-electrolyte interface investigated by FTIR. J Power Sources, 2001, 103(1): 10-17[DOI]

16 Ostrovskii D, Ronci F, Scrosati B, Jacobsson P. A FTIR and Raman study of spontaneous reaction occurring at the $\mathrm{LiNi}_{y} \mathrm{Co}_{(1-y)} \mathrm{O}_{2}$ electrode/non-aqueous electrolyte interface. J Power Sources, 2001, 94(2): $183-188[\mathrm{DOI}]$

17 Levi M D, Salitra G, Markovsky B, Teller H, Aurbach D, Heider U, Heider L. Solid-state electrochemical kinetics of Li-ion intercalation into $\mathrm{Li}_{1-x} \mathrm{CoO}_{2}$ : simultaneous application of electroanalytical techniques SSCV, PITT, and EIS. J Electrochem Soc, 1999, 146(4): $1279-1289[\mathrm{DOI}]$

18 Levi M D, Gamolsky K, Aurbach D, Levi M D, Gamolsky K, Aurbach D, Heider U, Oesten R. On electrochemical impedance of $\mathrm{Li}_{x} \mathrm{Co}_{0.2} \mathrm{Ni}_{0.8} \mathrm{O}_{2}$ and $\mathrm{Li}_{x} \mathrm{NiO}_{2}$ intercalation electrodes. Electrochim Acta, 2000, 45(11): 1781-1789[DOI]

19 Wang Z, Huang X, Chen L. Characterization of spontaneous reactions of $\mathrm{LiCoO}_{2}$ with electrolyte solvent for lithium-ion batteries. $\mathrm{J}$ Electrochem Soc, 2004, 151(10): A1641-A1652[DOI]

20 Gnanaraj J S, Thompson R W, Iaconatti S N, DiCarlo J F, Abraham K M. Formation and growth of surface films on graphitic anode materials for Li-ion batteries. Electrochem Solid-state Lett, 2005, 8(2): A128-A132[DOI]

21 Montella C. Review and theoretical analysis of ac-av methods for the investigation of hydrogen insertion I. Diffusion formalism. J Electrochem Chem, 1999, 462(2): 73-87[DOI]

22 Holzapfel M, Martinent A, Allion F, Holzapfel M, Martinent A, Alloin F, Le Gorrec B, Yazami R, Montella C. First lithiation and charge/discharge cycles of graphite materials, investigated by electrochemical impedance spectroscopy. J Electroanal Chem, 2003, 546: 41-50[DOI] 